\title{
Changes in Several Disease Parameters Including Abzymes and Hematopoietic Progenitor Colony Formation in Brain Inflammation and Demyelination
}

Doronin $\mathrm{VB}^{1}$, Korablev A ${ }^{2}$, Toporkova $\mathrm{LB}^{3}$, Aulova $\mathrm{KS}^{4}$, Buneva VN ${ }^{4,5}$, Budde ${ }^{6}$, Meuth $\mathrm{SG}^{7}$, Orlovskaya $\mathrm{IA}^{3}$, Popova $\mathrm{NA}^{2,5}$ and Nevinsky GA ${ }^{*, 5}$

${ }^{1}$ Novosibirsk Medical University, Ministry of Public Health of Russian Federation, Novosibirsk, Russia

${ }^{2}$ Institute Cytology and Genetics, Siberian Branch of the Russian Academy of Sciences, Novosibirsk, Russia

${ }^{3}$ Institute of Clinical Immunology, Siberian Branch of the Russian Academy of Sciences, Novosibirsk, Russia

${ }^{4}$ Institute of Chemical Biology and Fundamental Medicine, Siberian Branch of the Russian Academy of Sciences,

Novosibirsk, Russia

${ }^{5}$ Novosibirsk State University, Novosibirsk, Russia

${ }^{6}$ Westfälische Wilhelms-Universität, Institute of Physiology I, Münster, Germany

${ }^{7}$ Westfälische Wilhelms-Universität, Department of Neurology, Albert-Schweitzer-Campus 1, Münster, Germany

*Corresponding author: Nevinsky GA, Institute of Chemical Biology and Fundamental Medicine, Siberian Branch of the Russian Academy of Sciences, 8, Lavrentiev Ave., Novosibirsk, Russia, Tel: 73833635126, E-mail: nevinsky@niboch.nsc.ru

Citation: Doronin VB, Korablev A, Toporkova LB, Aulova KS, Buneva VN, et al. (2017) Changes in Several Disease Parameters Including Abzymes and Hematopoietic Progenitor Colony Formation in Brain Inflammation and Demyelination. J Neurol Neurol Disord 3(3): 302

Received Date: June 30, 2017 Accepted Date: August 02, 2017 Published Date: August 08, 2017

\begin{abstract}
Demyelination induced by cuprizone-is a widely used experimental model to analyze processes of re- and demyelination in the central nervous system (CNS). Here we used C57BL/6 mice; a model of experimental autoimmune encephalomyelitis (EAE) mimicking important aspects of human multiple sclerosis, to evaluate effects of cuprizone-dependent demyelination on different parameters associated with autoimmune inflammation. The treatment of mice with cuprizone leads to a significant decrease of several indexes characterizing spontaneous and myelin oligodendrocyte glycoprotein $\left(\mathrm{MOG}_{35-55}\right)$ - induced EAE: increased levels of titers of antiDNA and anti-MOG antibodies, proteinuria, the generation of abzymes hydrolyzing MOG, myelin basic protein (MBP), and DNA. In addition, spontaneous and MOG-induced EAE was associated with a specific reorganization of mice immune systems leading to significant changes in the level of proliferation and profile of differentiation of mice bone marrow hematopoietic stem cells (HSCs). The immunization of mice with cuprizone (0-40 days) results in a significant decrease in the size of the brain corpus callosum comparing with untreated mice, but only slightly changed level of proliferation and profile of differentiation of mice HSCs. Our data indicate that treatment with cuprizone is associated with demyelination, which is not associated with autoimmune processes.
\end{abstract}

Keywords: EAE model; C57BL/6 mice; Cuprizone-induced demyelination; Catalytic antibodies; Colony formation; Hematopoietic progenitors differentiation

\section{Introduction}

Multiple sclerosis (MS) is a demyelinating and inflammatory condition of the central nervous system (CNS) associated with perivascular infiltrates composed largely of T lymphocytes and macrophages. Despite the fact that the precise cause of MS remains unknown, numerous research data support the hypothesis of autoimmune mechanisms including the destruction of myelin playing a major role in the development of MS [1]. Evidence supports, that activation of CD4 ${ }^{+}$myelin-reactive T cells may be major mediator of MS. Several recent findings imply B cells important role and autoantibodies (auto-Abs) against autoantigens of myelin in the pathogenesis of MS [1-3].

Dual important role of auto-Abs was suggested: they may be potentially beneficial in lesion repair and vice versa be harmful in lesion formation [2]. In the cerebrospinal fluid (CSF) increased level of oligoclonal immunoglobulin G (IgG) and total Abs as well 
as accumulation of B cell in the CSF was observed. Several types of lesions are among the main lines of evidence for involvement of a humoral response in demyelination in MS patients [3,4]. Current evidence from animal models and clinical studies suggests that auto-Abs against myelin autoantigens are involved in antibody-mediated demyelination [3]. Auto-Abs against oligodendrocyte progenitor cell protein, which could reduce remyelination by impeding or eliminating these cells, may play a crucial role in MS immunopathogenesis [5].

Antibodies against transition states of chemical reactions (abzymes) catalyzing more than 200 different chemical reactions are novel enzymes; over the last years they have attracted much interest [6-9]. Natural catalytic abzymes hydrolyzing oligopeptides, proteins, nucleotides, RNA, DNA, and polysaccharides have been found in blood of patients with many autoimmune diseases [914]. Similarly to artificial abzymes against analogs of transition states of catalytic reactions, naturally occurring abzymes may be antibodies raised directly against enzyme substrates including DNA acting as haptens and mimicking transition states of catalytic reactions [9-14]. On the other hand, antiidiotypic Abs against catalytic centers of enzymes can also possess catalytic activities.

Natural abzymes with RNase and DNase activities in healthy humans and animals are usually absent [9-14]. In the blood of healthy mammals auto-Abs to various antigens, including different proteins, myelin basic protein, and DNA, are detectable but their titers vary significantly and all of them are usually catalytically inactive [10-16]. It was previously shown that the enzymatic activities of MBP-hydrolyzing, RNase, and DNase antibodies are easily detectable at the outset of autoimmune diseases, when concentrations of antibodies to different autoantigens are not yet remarkably increased and correspond to those in healthy donors [10-14]. It was shown that IgGs of MS patients are active in the hydrolysis of polysaccharides, RNA, and DNA and that specific hydrolysis of MBP and DNA is an intrinsic property of IgGs from CSF and sera of MS patients [17-27]. Interestingly, the CSF of MS patients contains Abs with MBP- and DNA-hydrolyzing activities; their relative activities are 50-60-fold higher than those of Abs from sera of the same patients [25-26]. In MS, MBP-hydrolyzing abzymes can attack MBP of the myelin-proteolipid sheath of axons. The known MS drug Copaxone (glatiramer acetate) was shown to be a specific inhibitor of abzyme-dependent MBP hydrolysis [27]. Thus, these catalytic antibodies can play an important and harmful role in pathogenesis of MS.

Many various abzymes were shown may be synthesized with a dramatically higher frequency and activity in autoimmune mouse strains than in control non-autoimmune conventionally used mouse strains [28,29]. It was shown, that in SLE anti-DNA Abs are mainly directed against nucleosomal complexes of histones with DNA, appearing during apoptosis as a result of internucleosomal cleavage [30].

There is a viewpoint that autoimmune diseases originate from different defects in hematopoietic stem cells (HSCs) [31]. It was recently shown that the immune system reorganization during the spontaneous development of a deep SLE-like pathology in MRL-lpr/lpr mice is strongly associated with changes in the differentiation profile of bone marrow HSCs in combination with the production of DNase, ATPase, and amylase abzymes [32-34]. The healthy mice treatment with DNA also leads to the production of DNase abzymes; however, it is associated mostly with increase in proliferation of lymphocytes and suppression of lymphocytes apoptosis in various organs (especially the spleen), but not with remarkable changes in the bone marrow cells differentiation [3234]. Taking this into account, we have suggested that patients with MS may have similar changes in profile of differentiation of bone marrow HSCs as well as in the level of lymphocyte proliferation. In this case, MS patients can produce not only typical Abs to DNA and MBP, but also abzymes hydrolyzing DNA and proteins.

Several various mice models of EAE mimicking a particular facet of human MS are available [35]. C57BL/6 inbred mice seem to be prone to develop EAE based on the spontaneous generation of potentially harmful abzymes and a specific stem cell differentiation pattern. These natural tendencies are boosted by immunization with myelin proteins. Therefore, C57BL/6 is regarded as a potential mouse model of spontaneous EAE. Recently we have used $\mathrm{MOG}_{35-55}$ for acceleration of EAE [36]. It was shown, that during development of spontaneous and MOG-induced EAE, the mice immune system specific reorganization leads to a specific condition associated with the generation of abzymes hydrolyzing MOG, MBP, and DNA, as well as to increased proteinuria, changes in differentiation and proliferation of mice bone marrow HSCs and lymphocytes. The strongest alterations of these indexes were found in the case of MOG-induced EAE.

Since cuprizone has selective specificity against oligodendrocytes, demyelination induced by cuprizone is usually widely used as experimental model to investigate the myelin pathology in MS [37,38]. Acute demyelination usually observed after mice treatment with cuprizone is associated with oligodendrocytes apoptosis, phagocytosis of myelin sheaths and activation of microglia [39,40]. Demyelination processes causes by cuprizone allover the brain and the corpus callosum is the most affected structure. In addition, distinct foci of demyelination are also found in the cerebellum, putamen, hippocampus, and the ventral part of caudal nuclei.

Abzs activities are usually detectable even at the stage of pre-disease, when there is no visible markers of SLE, MS or other AI diseases and changes in proteinuria, and the titres of Abs against DNA, myelin basic protein (MBP) or other antigens are within the typical ranges of these indicators for healthy human and mice. Thus, detectable and especially high level of Abz activities can be considered as an indicator even of the pre-disease (beginning of the pathology) and obvious pathology conditions of spontaneous or specifically induced autoimmune diseases [9-14]. Therefore it was interesting to analyze how cuprizone can influence on defects of immune system even at outset of spontaneous development of EAE and following production of different catalytic antibodies showing the development of autoimmune reactions resulting from changes in the profile of stem cells differentiation in mice bone marrow. 
As mentioned above, during development of EAE the specific reorganization mice immune system is associated with the generation of abzymes hydrolyzing MOG, MBP, DNA, and other antigens as well as with demyelination of the CNS. The mice treatment with cuprizone also leads to the demyelination of the CNS. Therefore it was interesting to compare the influence of cuprizone and activator (MOG) of EAE development on demyelination processes, changes in the cell differentiation profiles and the development of abzymes with different activities.

Here we immunized C57BL/6 mice with MOG and treated with cuprizone for comparing some biochemical markers of EAE pathology (titers of Abs to MOG and to DNA, proteinuria) as well as relative activities of MBP-, MOG-, and DNA-hydrolyzing mouse IgGs. In addition, changes in parameters of differentiation of bone marrow HSCs before and after mice treatment with MOG or with cuprizone were also compared. The changes of these parameters in the cuprizone model were compared with spontaneous and MOG-induced EAE.

\section{Materials and Methods}

\section{Chemicals}

All proteins, chemicals, Superdex 200 HR 10/30 column, and Protein G-Sepharose, were purchased from Sigma or GE Healthcare. Cuprizone (bis(cyclohexanone)oxaldihydrazone) was obtained from Sigma-Aldrich, $18.5 \mathrm{kDa}$ human MBP from RCMDT

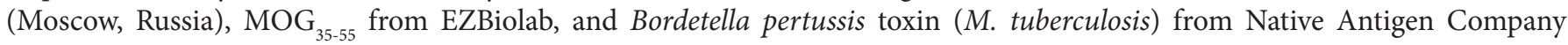
(Oxfordshire, UK). All preparations were free from nucleic acid, oligosaccharides, lipids, and other possible contaminants.

\section{Experimental Animals}

C57BL/6 inbred 3 months aged autoimmune mice (males; $22 \pm 2.0 \mathrm{~g}$ at the beginning of experiments) were housed in colonies under standard pathogen-free conditions at the Institute of Cytology and Genetics (ICG) mouse breeding facility having a system for protection from bacterial and viral infections. All experiments with mice were carried out in accordance with protocols of Bioethical Committee of the ICG, which protocols correspond to Bioethical Committee of the Siberian Branch of the Russian Academy of Sciences and the recommendations of the European Committee for the humane principles of work with experimental animals (European Communities Council Directive 86/609/CEE). Bioethical Committee of the ICG has approved our study in accordance with European Communities Council Directive 86/609 guidelines.

\section{Immunization of Mice with MOG and Treatment with Cuprizone}

Mice immunization with MOG was carried out using MOG $_{35-55}$ and Pertussis toxin (Mycobacterium tuberculosis) according published protocol as described in [36,41].

Demyelination was carried out using $0.33 \%$ water solution of cuprizone. Cuprizone was first solved in water at $60{ }^{\circ} \mathrm{C}$, then cooled and filtered. The solution was given to mice instead of drinking during 39 days, changing the solution was carried out every three days.

The relative mice weight and level of proteinuria (relative concentration of total protein in the urine, $\mathrm{mg} / \mathrm{ml}$ ) were analyzed as in [34]. Concentration of urine protein was estimated by the Bradford assay with a bovine serum albumin standard. For different experiments including Abs purification and enzymatic activity analysis $0.7-1 \mathrm{ml}$ of the blood (after mice decapitation) were collected using standard protocols [36].

\section{ELISA of Anti-Protein and Anti-DNA Abs}

Concentration of Abs against MOG was estimated by standard ELISA (plasma was diluted 50-fold). Serum anti-DNA Abs concentrations were measured by standard ELISA plates with immobilized double-stranded DNA (plasma was diluted 100fold) as in [34,36]. After a consecutive the blood serum samples treatment with rabbit specific anti-mouse Abs conjugating with horseradish peroxidase, the reaction mixtures were incubated with $\mathrm{H}_{2} \mathrm{O}_{2}$ and tetramethyl benzidine. The reaction was stopped by addition of $\mathrm{H}_{2} \mathrm{SO}_{4}$ and the solutions optical densities $\left(\mathrm{A}_{450}\right)$ were measured using a Uniskan II plate reader (MTX Lab Systems, USA). The relative concentrations of Abs against MOG and DNA in the samples were expressed as a difference in the relative absorption at $450 \mathrm{~nm}$ between control and experimental samples; controls with MOG or DNA, but without serum samples and with Abs not interacting with DNA and MOG showed the same results.

\section{IgG Purification}

Electrophoretically homogeneous mouse IgGs were purified using sequential chromatography of the serum proteins on Protein G-Sepharose and following FPLC gel filtration according to [22-24,32-34]. In order to protect IgGs from viral and bacterial contaminations, the preparations were filtered through Millex syringe-driven filter units $(0.1 \mu \mathrm{m})$ and kept in sterilized tubes. Incubation of bacterial standard medium with stored IgGs did not result in the colonies formation. SDS-PAGE assay of the IgG fractions for homogeneity was done under non-reducing conditions in 4-15\% gradient gels; for polypeptide separation it was carried out in a reducing gel $(0.1 \%$ SDS and $10 \mathrm{mM}$ dithiothreitol); the proteins and polypeptides were revealed by silver staining 
as in [22-24,32-36]. To exclude possible artefacts due to admixtures of canonical enzymes, IgGs were separated by standard SDSPAGE and their DNase and protease activities were revealed using a gel assay as in [22-24,32-36]. These enzymatic activities were detected only in the band of intact IgGs, and there was no observed other protein peaks as well as peaks of proteolytic or DNase activities.

\section{DNA-Hydrolyzing Activity Assay}

DNase activity was analyzed according to described methods [34,36]. The reaction mixtures $(20 \mu \mathrm{l})$ containing $20 \mathrm{mM}$ Tris$\mathrm{HCl}, \mathrm{pH}$ 7.5, $20 \mu \mathrm{g} / \mathrm{ml}$ supercoiled (sc) pBluescript, $5 \mathrm{mM} \mathrm{MgCl}, 20 \mathrm{mM} \mathrm{NaCl}, 1 \mathrm{mM}$ EDTA, and 0.03-0.2 mg/ml of Abs, and were incubated at $37^{\circ} \mathrm{C}$ for $2-12 \mathrm{~h}$. The products of cleavage were analyzed using electrophoresis in $0.8 \%$ agarose gels. Ethidium bromide-stained gels were photographed and the films were scanned. The relative activities of IgGs were estimated from the scans (Gel-Pro Analyzer v9.11) as a relative percentage of DNA corresponding to band of non-hydrolyzed scDNA and its relaxed form; a distribution of DNA between these bands in the case of incubation of pBluescript in the absence of Abs (control experiments) was taken into account. All initial rates were determined using the linear regions of over time course and dependencies of the rates on Ab concentration (15-40\% of DNA hydrolysis). A complete scDNA transformation to nicked DNA was taken for $100 \%$ of the activity. If the activity was relatively low $(<5-10 \%$ of scDNA disappearance), the time of incubation depending on the sample was increased to 3-12 h. If the scDNA degradation exceeded 50\%, depending on the sample analyzed the concentration of IgGs was decreased $~ 2-100$ fold. Finally, the relative activities (RAs) of Abs were normalized to the same standard conditions.

\section{Protease Activity Assay}

For analysis of IgG' MBP- or MOG-hydrolyzing activities the reaction mixtures (10-40 $\mu \mathrm{l}$ ) contain $20 \mathrm{mM}$ Tris- $\mathrm{HCl}$ (pH 7.5), 0.7 $\mathrm{mg} / \mathrm{ml} \mathrm{MBP}$ or MOG and 0.01-0.2 mg/ml of IgGs; they were incubated at $37{ }^{\circ} \mathrm{C}$ for $2-20 \mathrm{~h}$. The cleavage products of MBP and MOG were analyzed using SDS-PAGE in $12 \%$ or $4-15 \%$ gradient gels with following Coomassie R250 staining. The gels were scanned and quantified using GelPro v3.1 software. The relative activities of IgGs were estimated from a decrease in the percentage of initial MBP or MOG (100\%) converted to their various hydrolyzed forms comparing to control MOG or MBP incubated without Abs. All measurements (initial rates) were performed using the conditions of the pseudo-first order of the reaction within the linear regions of the dependencies of substrates hydrolysis on IgG concentration and the time course (15-40\% of MBP).

\section{Analysis of Bone Marrow Progenitor Cells in Culture}

Bone marrow was flushed out of the mouse femurs. For evaluation of the colony-forming ability, of bone marrow cells, $2 \times 10^{4}$ cells (4 dishes per mouse) were incubated using a standard methylcellulose-based M3434 medium for mouse cells (StemCell Technologies, Canada) containing erythropoietin (EPO), stem cell factor, and IL-6, and interleukin (IL)-3. Colonies (granulocyticmacrophagic colony-forming unit [CFU-GM], erythroid burst-forming unit-early erythroid colonies [BFU-E], erythroid burstforming unit-late erythroid colonies [CFU-E], and granulocytic-erythroid-megacaryocytic-macrophagic colony-forming unit [CFU-GEMM]) were scored after 14 days of incubation at $37{ }^{\circ} \mathrm{C}$ and $5 \% \mathrm{CO}_{2}$ in a humidified incubator as in [32-36].

\section{Statistical Analysis}

The results are reported as the mean \pm standard deviation of at least 3-4 independent experiments for each mouse, averaged over 7 different animals. Differences between the samples were analyzed by Student's $t$-test, $p \leq 0.05$ was considered to be statistically significant.

\section{Results}

\section{Experimental Groups of Mice}

Here we have used three experimental groups of C57BL/6 (7 mice for each time point) and control CBA mice:

1) Control untreated C57BL/6 mice,

2) Cuprizone treated $\mathrm{C} 57 \mathrm{BL} / 6$ mice,

3) MOG-immunized C57BL/6 mice.

To achieve mice EAE, we used in this study $\mathrm{MOG}_{35-55}$. Previous studies, using different selected peptides, have indicated that $\mathrm{MOG}_{35-55}$ peptide is an encephalytogenic epitope in C57BL/6 mice [42]. It was shown, that the substitutions of Arg at positions 41 and 46 with Ala results in peptide analogues that reduce the severity of MOG-induced EAE clinical symptoms in C57BL/6 mice $[43,44]$. Different chemical modifications of MOG including its nitration and other modifications, as well as its association with vitamin D prevents EAE development [45-47].

After immunization with MOG, EAE mice usually develop first clinical symptoms at 5-7 days, but the maximum stage of this disease is observed at 14-16 days after the treatment $[41,48]$. We have analyzed possible changes in different biochemical and immunological parameters, relative weight from zero time ( 3 months of age, control) and following 40 days in cuprizone-treated C57BL/6 mice (Figure 1A) in comparison to MOG-treated mice (performed similarly to [36]). The average mice weight loss was revealed after several days in cuprizone- and MOG-treated comparing with untreated control mice (Figure 1A). Thereafter the weight gain in cuprizone-treated mice was remarkably slower than that for untreated and MOG-treated animals (Figure 1A). 
A

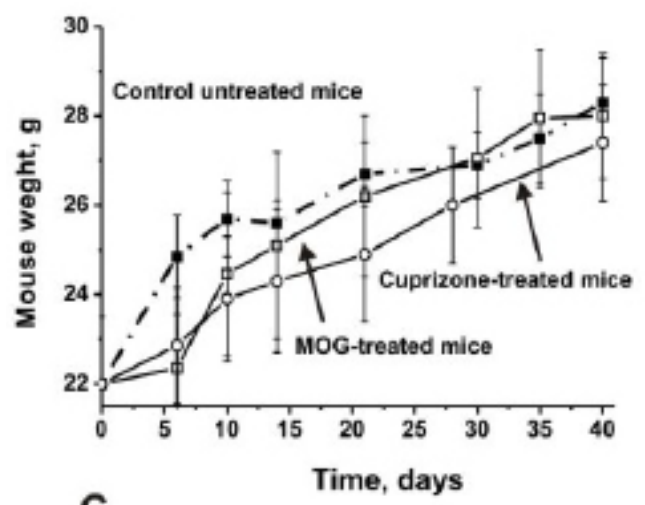

C

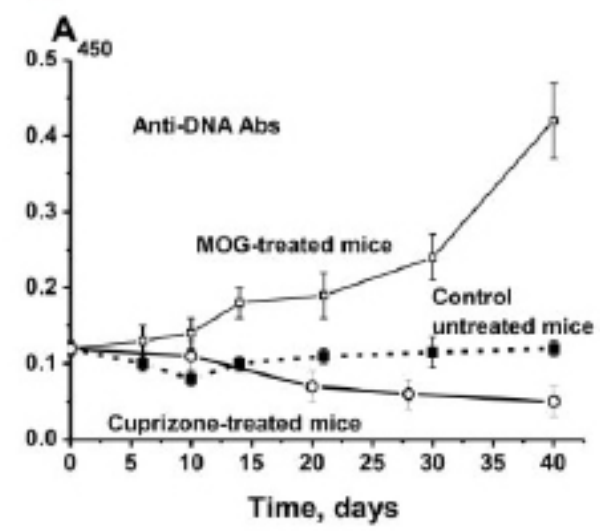

B

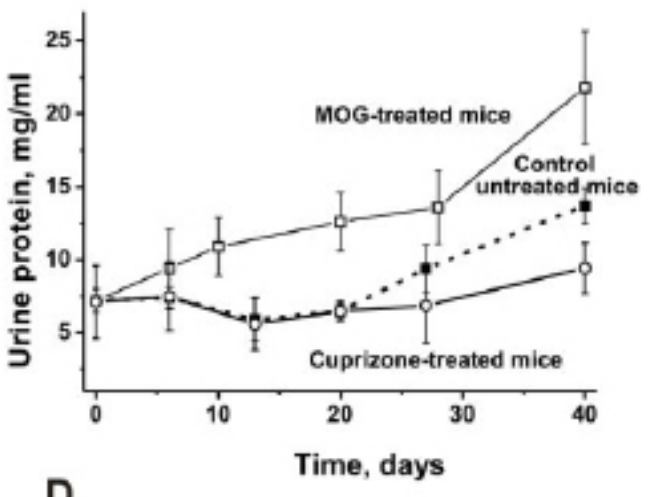

D

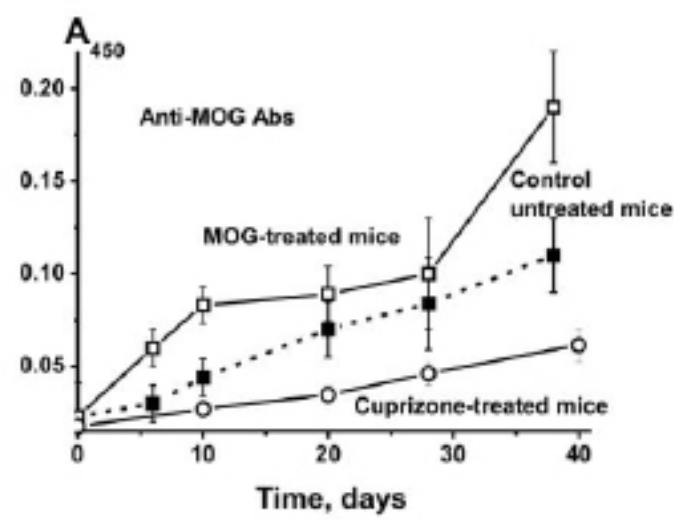

Figure 1: Changes in body weight of C57BL/6 mice over time (A), relative concentration of urine protein (B), titers of Abs to DNA (C), and to MOG (D) in mice treated with cuprizone, untreated and treated with MOG. Anti-MOG and anti-MBP antibody concentrations in the sera of C57B2/6 mice were measured by ELISA (plasma was diluted 50-fold). The concentration of plasma anti-DNA Abs was determined using standard ELISA plates with immobilized double-stranded DNA (plasma was diluted 100-fold)

Different autoimmune mice models including MRL-lpr/lpr, usually demonstrate appearance of visible symptoms correlating well with proteinuria (concentration of protein in urine $\geq 3 \mathrm{mg} / \mathrm{ml}$ ) [32-34]. Non-autoimmune BALB and CBA control mice at least during 7 months have no proteinuria $(0.1-0.12 \mathrm{mg} / \mathrm{ml})$ [32-34]. Autoimmune MRL-lpr/lpr healthy mice before spontaneous development of deep SLE are usually demonstrated relatively low proteinuria $(0.38 \mathrm{mg} / \mathrm{ml})$ [32-34]. At the same time, C57BL/6 is usually characterized by a significantly increased level of proteinuria (up to $10-12 \mathrm{mg} / \mathrm{ml}$ ) [49]. The analysis of time-dependent changes in proteinuria of MBP-, and cuprizone-treated as well as untreated C57BL/6 mice were carried out (Figure 1B). For all groups consisting of seven mice the average urine protein concentration at zero time ( 3 months of age) was $7.2 \pm 0.8 \mathrm{mg} / \mathrm{ml}$. It was nearly the same until day 20 and then increased at 40 days for control and cuprizone-treated mice respectively to 12.0 and $9.4 \mathrm{mg} /$ $\mathrm{ml}$. For mice with accelerated EAE due to their treatment with MOG the proteinuria increases significantly faster and at 40 days it was significantly higher: $21.8 \pm 3.9 \mathrm{mg} / \mathrm{ml}$ (Figure $1 \mathrm{~B}$ ). Thus, the treatment of mice with cuprizone results in a statistically significant decrease $(P<0.05)$ of the proteinuria by 1.3 -and 2.3 -fold compared to untreated and MOG treated EAE mice, respectively.

\section{Determination of the Relative Content of Anti-DNA and Anti-MOG Antibodies}

The blood of MS patients usually contain anti-MBP and anti-DNA Abs [17,18,22-24]. The relative anti-DNA Abs concentrations for Balb and CBA mice during 2-7 months of age and MRL-lpr/lpr healthy mice (2-3 months of age) are typically in the range of 0.03-0.04 $\mathrm{A}_{450}$ units [34]. We have estimated the relative concentration of anti-DNA Abs in the blood of C57BL/6 mice using the same test (1C). The average concentration of anti-DNA Abs at three months of age in the case of untreated C57BL/6 mice was 0.12 $\mathrm{A}_{450}$ units and it was nearly the same during 40 days of the analysis (Figure 1C). Interestingly, after mice treatment with cuprizone there was a slow decrease in the concentration of anti-DNA Abs and at 40 days it became to be 2.4 -fold lower $(P<0.05)$ than at the beginning. The relative anti-DNA Abs concentration for MOG-treated mice at 40 days was 3.5-and 8.4-fold higher than that respectively for untreated and cuprizone-treated mice (Figure 1C). Thus, the treatment of C57BL/6 mice with MOG stimulated the generation of anti-DNA Abs, which is a specific characteristic of several different autoimmune diseases including SLE and MS, while cuprizone feeding results in a significant decrease of this index [10-14].

Figure 1D shows over time changes in anti-MOG antibodies in the sera of cuprizone-, MOG-treated and untreated C57BL/6 mice. The average relative concentration of antibodies against MOG in seven untreated mice was significantly $(P \leq 0.05)$ increased during $\sim 40$ days by a factor of $\sim 4.8$ in nearly linear fashion. Cuprizone decreased the formation of anti-MOG antibodies $\sim 2$-fold, while immunization with MOG results in the increase of the average anti-MOG Abs concentration comparing to untreated mice $\sim 1.7$-fold (Figure 1D). 


\section{Determination of the Relative Proteolytic and Dnase Activities of Antibodies}

To search for catalytic antibodies, IgGs were obtained from the sera of individual mice by affinity chromatography on Protein G-Sepharose using special condition to remove non-specifically bound proteins as in [22-24,32-34]. IgGs were then subjected to FPLC gel filtration. For analysis of DNase and protease Ab activities, individual preparations from various animals and mixtures of equal amounts of homogeneous IgGs from the sera of MOG- (MOG-IgG ${ }_{\text {mix }}$ ), cuprizone-treated (cupr-IgG mix $_{\text {mix }}$ ) and untreated (untr$\mathrm{IgG}_{\text {mix }}$ ) mice during 40 days after treatment were used. The electrophoretic homogeneity of MOG-IgG ${ }_{\text {mix }}$, cupr-IgG ${ }_{\text {mix }}$, and untr$\mathrm{IgG}_{\text {mix }}(150-\mathrm{kDa})$ was confirmed by silver staining after standard SDS-PAGE. All IgGs demonstrated a single band under nonreducing conditions (for example, Figure 2A) and two bands corresponding to the $\mathrm{H}$ and $\mathrm{L}$ chains in reducing conditions (Figure 2B). It was previously shown, that Abs from non-autoimmune control BALB and CBA mice (3-7 months of age) have no detectable DNase, MOG-, and MBP-hydrolyzing activities, while IgGs from MOG-treated and untreated C57BL/6 mice demonstrated high activity in the hydrolysis of these substrates [32-34,36]. In this study we confirmed previously obtained results; taking into account the deviation of the activity values for seven MOG-treated mice of each group, the average relative activity of Abs in this study were not differ from those obtained in [36]. After C57BL/6 mice treatment with cuprizone IgGs were also active in the hydrolysis of MOG and DNA.

Based on a previously established set of strict criteria we have concluded that the observed hydrolysis of MBP, MOG, and DNA is intrinsic property of IgGs from the blood of cuprizone-treated and untreated C57BL/6 mice and is not due to any admixtures of canonical proteases or nucleases [10-14].

The most important criteria were checked: a) electrophoretic homogeneity of IgGs (Figure 2A and 2B); b) FPLC gel filtration of cupr-IgG ${ }_{\text {mix }}$ performed using conditions of acidic shock ( $\mathrm{pH} 2.6$ ) does not lead to a disappearance of the catalytic activities, and the peaks of DNase and proteolytic activities exactly coincided with peaks of IgGs (Figure 2C); c) Sepharose bearing Abs against mouse IgG light chains binds completely these activities and they were eluted from the sorbent with acidic buffer. To exclude

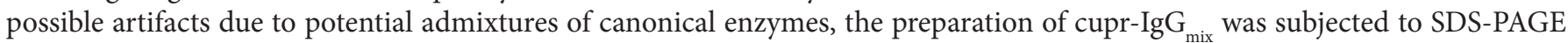
and its proteolytic and DNase activities were checked after proteins extraction from the separated gel slices (Figure 2D and 2E). IgG enzymatic activities were found only in the size of intact IgGs. SDS dissociates usually any protein complexes. Electrophoretic mobility of known relatively small canonical proteases and DNases (28-32 kDa) cannot coincide with that of intact IgGs (150 $\mathrm{kDa}$ ). Therefore, the reveling of DNase and protease activities in the gel region corresponding to only intact IgGs together with the absence of any other bands of proteins and enzymatic activities (Figure 2), guaranties direct evidence that even after C57BL/6 mice treatment with cuprizone IgGs possess analyzed activities.

Next we have estimated the relative DNase and protease activities (RAs) of individual IgG preparations from the cuprizonetreated animals and untreated control mice (each group of 7 mice) during 0-40 days after the treatment. Figure 3 shows the RAs corresponding to IgG mixtures in the hydrolysis of MBP (A) and DNA (B). To quantify the DNA-, MBP-, and MOG-hydrolyzing activities of Abs in the case of different mouse groups, we estimated a concentration of each individual IgG preparation that converted MOG and MBP to their cleavage products (no more than 40\%) and scDNA plasmid to its relaxed form without remarkable accumulation of linear or fragmented DNA (no more than $40 \%$ of initial DNA). All initial rates were measured using the linear regions of the time courses and Abs concentration curves. Therefore, the estimated RAs for all IgG preparations were normalized to standard conditions. The averaged data on enzymatic activities for 7 mice of each group are summarized on Figure $3 \mathrm{C}, 3 \mathrm{D}$ and $3 \mathrm{E}$.
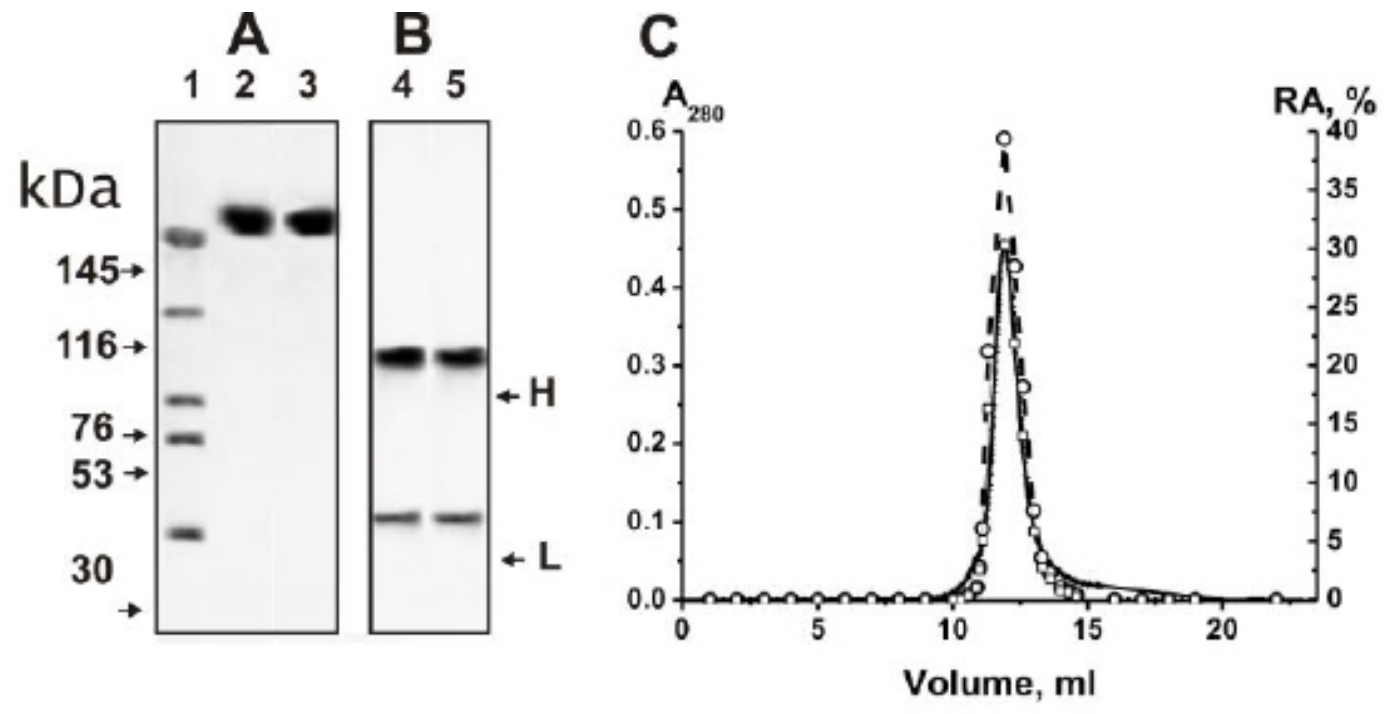


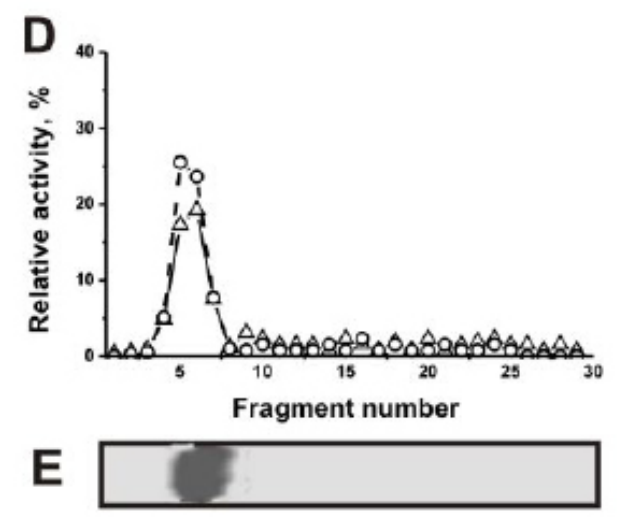

Figure 2: SDS-PAGE analysis of the homogeneity of untr- $\operatorname{IgG}_{\text {mix }}$ and cupr- $\operatorname{IgG}_{\text {mix }}(7 \mu \mathrm{g})$ under non-reducing (A, lanes 2 and 3 , respectively) and reducing (B, lanes 4 and 5) conditions followed by silver staining; the arrows (A, lane 1) indicate the positions of molecular mass markers. FPLC gel filtration of cupr-IgG $\mathrm{Imix}_{\text {m }}$ on a Superdex 200 column in an acidic buffer ( $\left.\mathrm{pH} 2.6\right)$ after Abs incubation

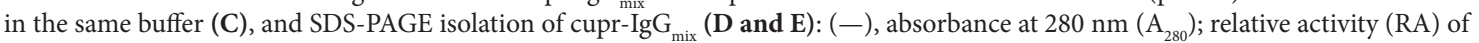
DNA (o) and MBP $(\Delta)$ hydrolysis. The relative DNase- and MBP-hydrolyzing activity (\%) was revealed using the extracts of 2-3 mm fragments of one longitudinal slice of the gel (D). Panel E shows the position of IgGs after electrophoresis. A complete hydrolysis of substrates for $24 \mathrm{~h}$ was taken for $100 \%$ (C, D). The error in the initial rate determination from two experiments in each case did not exceed 7-10\%. For other details see Methods

During 40 days of the experiment, control non-treated mice showed a nearly linear average statistically significant increase in DNase (5.4-fold), MBP (1.7-fold), and MOG- (1.8-fold) hydrolyzing activities (Figure 3). Mice treatment with cuprizone did not result in any statistically significant change in DNase activity (average of mean values $4.1 \pm 0.1 \%$ ) from 0 to 40 days of the experiment (Figure 3C), while the treatment with MOG resulted in the increase of this activity 24.4 -and 9.8 -fold at 22 and 40 days, respectively. The relative MBP-hydrolyzing activity of IgGs in cuprizone-treated mice did not change remarkably during 0-40 days, while for MOG-treated mice the activity increased by a factor of 3.2 in comparison to day 0 (Figure 3D). At the late stage of EAE (40 days), the RA of IgGs from mice treated with cuprizone in the hydrolysis of MOG was 1.3-fold lower, while for MOG-treated mice 3.2-fold higher comparing with the beginning of the experiment (Figure 3E). Thus, the mice treatment with cuprizone results in a decrease in the production of anti-DNA, anti-MBP, and anti-MOG antibody concentrations and their relative activities in the hydrolysis of MOG and DNA did not remarkably changed in comparison with their initial activities at zero time, while during spontaneous development of EAE and especially after treatment of mice with MOG there were significant increases in these activities (Figure 1 and 3 ).

\section{Number of days}

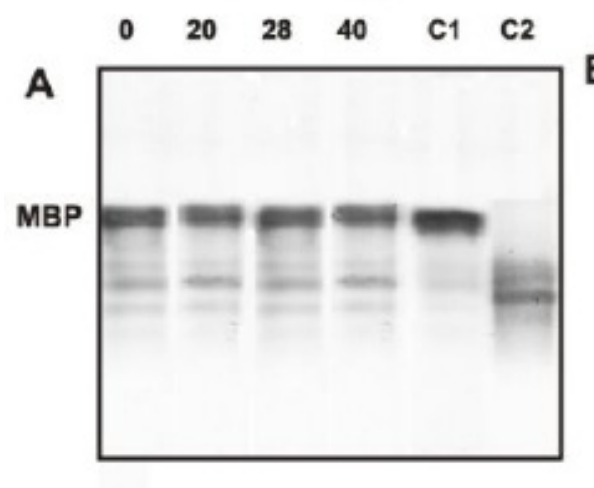

B Number of days

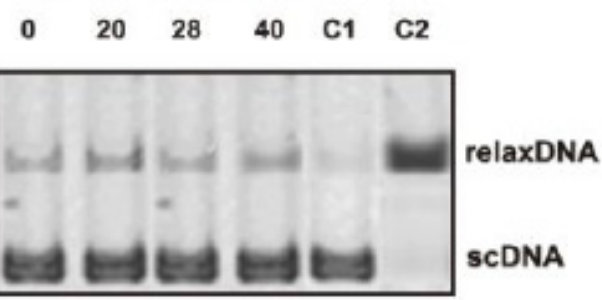

C

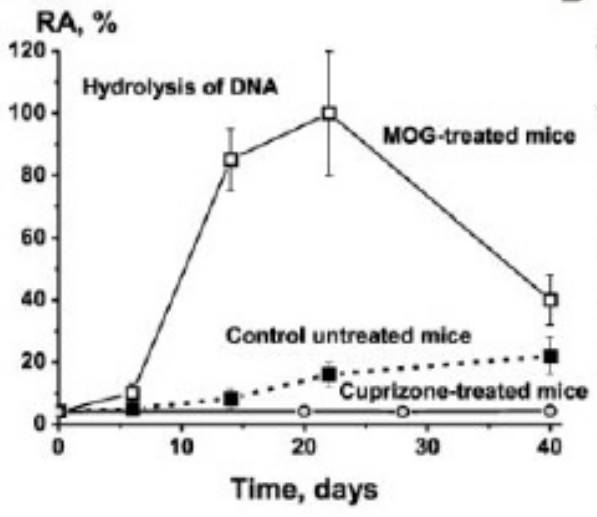

D

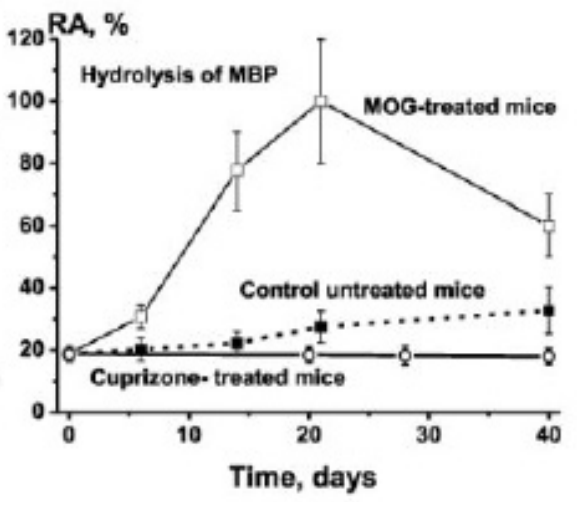




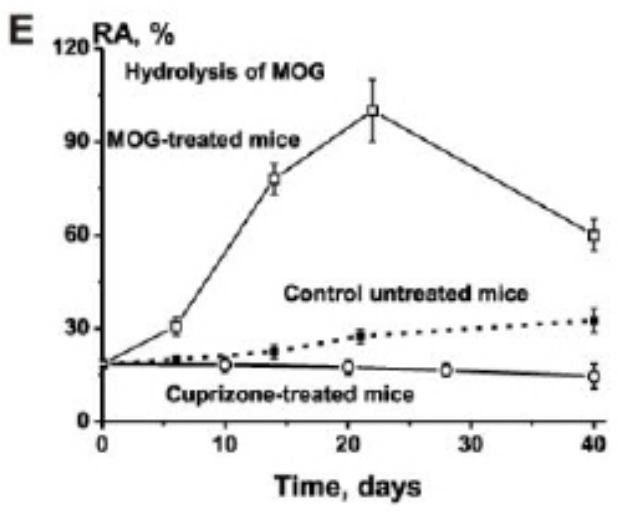

Figure 3: Analysis of the relative MBP-hydrolyzing activity by SDS-PAGE (A) and DNase activity by agarose electrophoresis (B) of IgGs from the blood plasma of mice treated with cuprizone at 0-40 days after their immunization; equimolar mixture of IgGs of the cuprizone-treated groups of mice (each group of 7 mice) after 0, 20, 28, and 40 days after the treatment. Lanes $\mathbf{C 1}$ and $\mathbf{C 2}$ correspond respectively to these substrates incubated in the absence of IgGs and in the presence of IgGs from control MOG-treated mice, respectively. MBP $(0.5 \mathrm{mg} / \mathrm{ml})$ and scDNA $(20 \mu \mathrm{g} / \mathrm{ml})$ were incubated in the absence or in the presence of $0.1 \mathrm{mg} / \mathrm{ml} \mathrm{IgGs}$ for $9 \mathrm{~h}$. Changes in the relative average activity of IgGs from the blood plasma of untreated and MOG-treated mice in the hydrolysis of DNA (C), MBP (D) and MOG (E) over time; average relative activity (RA) of IgGs corresponding to 7 individual mice for each group is given. The error in the initial rate determination from two experiments in the case of every mouse of each group did not exceed 7-10\%. For other details see Methods

After mice immunization with MOG the maximum stage of EAE disease (according to the literature) is usually manifested at 14-18 days [41]. Interestingly, the relative concentrations of anti-DNA, anti-MBP, and anti-MOG antibodies gradually increase from 0 to 40 days (Figure 1C and 1D), while the maximal enzymatic activities of IgGs are observed at 18-22 days followed by a significant decrease (Figure 3C and 3D). Our results show that transition from early to late EAE stages can lead to a decrease in the production of different abzymes as a result of a switch of the mouse immune system towards the synthesis of Abs without catalytic activity. These findings are in agreement with the increase in RAs of abzymes against various antigens at the onset of autoimmune diseases and a significant decrease in their catalytic activities during remissions [9-14]. At the same time, the treatment of mice with cuprizone results in the decrease in parameters which are indicators of development of autoimmune processes, namely the concentrations of urine proteins (Figure 1B), anti-DNA, anti-MOG antibodies concentrations (Figure 1C and 1D), and all enzymatic activities (Figure 3C and 3E). In addition, an increase in the weight of mice treated with cuprizone is slowed down in comparison with untreated mice (Figure 1A). Overall, cuprizone suppresses autoimmune processes in autoimmune C57BL/6 mice. The question is concerning a possible mechanism of this effect of cuprizone on mice immune system. It is known, that a hallmark of MS is partial demyelination of the patients brains leading to the formation of plaques which are detectable by MRI. As mentioned above, cuprizone can cause fast demyelination processes in several regions of the brain, the most affected structure is the corpus callosum $[39,40]$. Therefore, we have compared demyelination in the corpus callosum of cuprizone-treated and

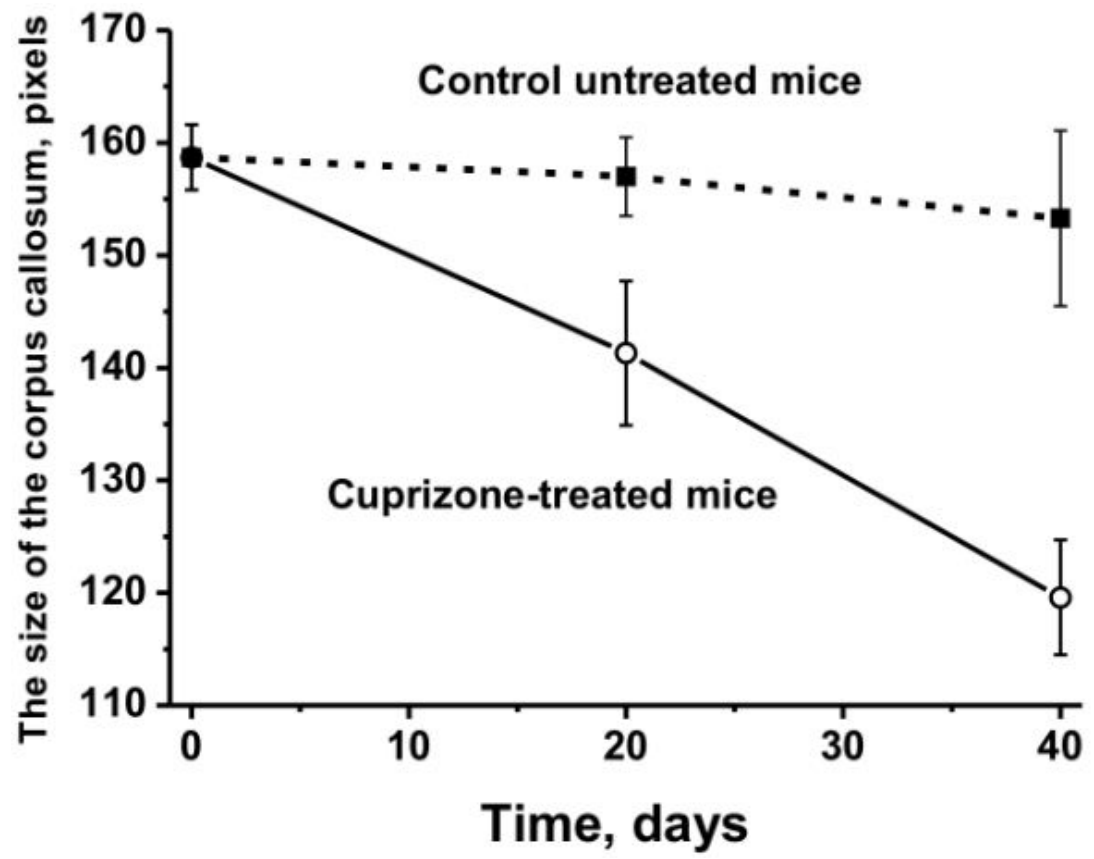

Figure 4: A comparison of demyelination of the corpus callosum from untreated and cuprizone-treated mice. The size of the corpus callosum of untreated and cuprizone-treated mice was analyzed by MRI. 
untreated mice (Figure 4). We found, that the size of the corpus callosum of untreated mice decreases statistically insignificant from 0 to 40 days by $3.5 \%$, while after treatment with cuprizone resulted in a $25 \%$ decrease $(P<0.05)$. Thus, specific cuprizone effects on the mice can be due not only demyelination of the brain, but also alteration of Abs expression.

\section{Colony Formation of Hematopoietic Progenitors}

First we have analyzed potential changes of the sum of all colony units (CFU-E + BFU-E + CFU-GEMM + CFU-GM) for untreated as well as cuprizone- and MOG-treated mice over time (Figure 5A). The level of proliferation of the summed hematopoietic

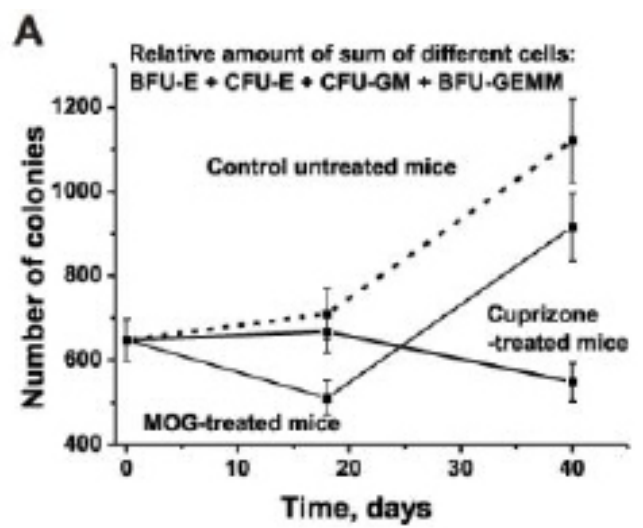

B
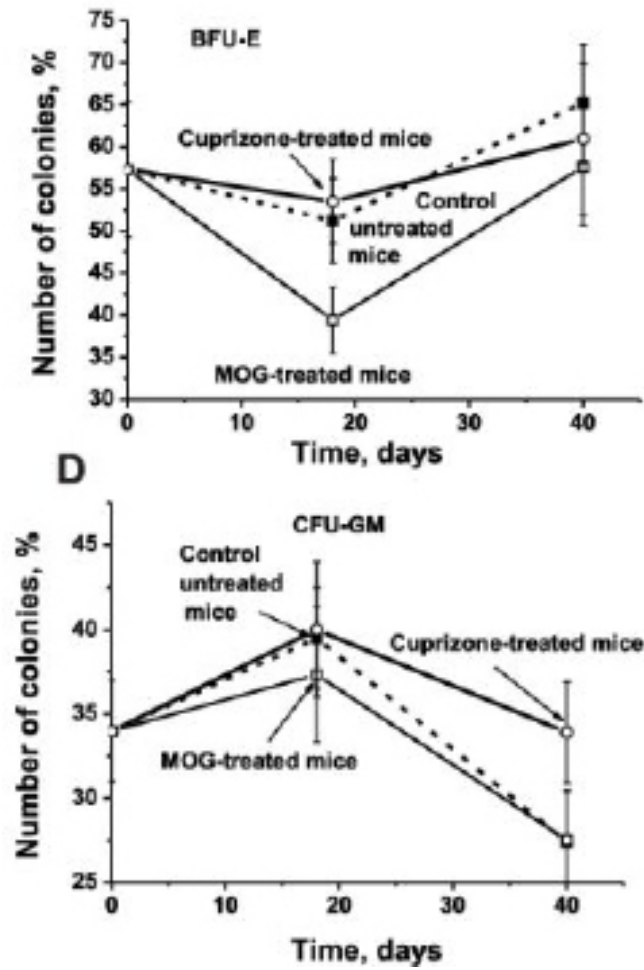

C
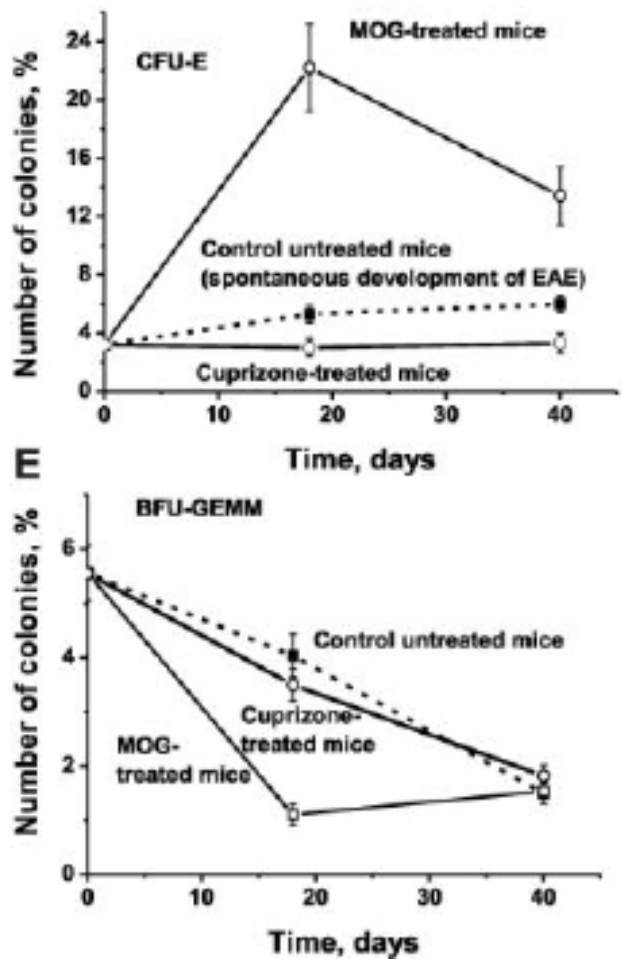

Figure 5: Changes over time of four types of colony (BFU-E + CFU-E + CFU-GM + CFU-GEMM) formation of untreated as well as cuprizone- and MOG-treated mice (A). Change of the relative percent (sum of four types of colonies was taken for 100\%) of BFU-E (B), CFU-E (C), CFU-GM (D), and BFU-GEMM (E). For other details see Methods

progenitor colonies in the case of untreated mice initially slowly increased from 0 to 22 days followed by a statistically significant 1.7 -fold rise at 40 days in comparison to day zero (Figure 5A). The treatment of mice with MOG leads to 1.3 -fold $(P<0.05)$ decrease in the summed number of colonies at 22 days, but then it increases at 40 days approximately by a factor of 1.4 in comparison with the initial level. Interestingly, the treatment of mice with cuprizone does not influence the level of colonies formation up to 22 days, but it decreases $\sim 1.2$-fold to 40 days of the experiment. Thus, cuprizone generally slows down the formation of cell colonies in comparison with spontaneous and MOG-induced development of EAE. Next we assessed the level of colonies formation by the four types of hematopoietic precursors separately.

In order to estimate the relative number of colonies of four different types, we have determined a relative percent of every type of the colony units with respect to their total number taken as $100 \%$ (Figure 5A). Figure 5B and 5E show the changes in percentage of the specific types of colonies. 
For control untreated mice the proportion of BFU-E colonies was increased during 0-40 days from 57.3 to $65.2 \%$, CFU-E colonies from 3.2 to $6.0 \%$. CFU-GM colonies increase from 34 to $40 \%$ (22 days) and then decreased to $27.4 \%$ at 40 days (Figure $5 \mathrm{~B}$ and 5D). Interestingly, the average percent of CFU-GEMM colonies during 40 days was reduced from 5.6 to $1.5 \%$ (Figure 5E). Thus, the profile of differentiation of bone marrow progenitors is significantly changed during spontaneous development of mouse EAE.

For cuprizone-treated mice the percent of BFU-E first decreases from 57.3 to 53.5 and then it increases to 60.9\% (Figure 5B), while the percent of CFU-GM first increases from 34 to $39.5 \%$ and then it decreases to $27.4 \%$, thereby revealing some similarities to untreated mice (Figure 5B and 5D). The time courses for CFU-GEMM colonies for untreated and cuprizone-treated mice were nearly identical and revealed a significant decrease in the level of colony formation during 40 days from 5.6 to 1.5 and $1.8 \%$, respectively (Figure 5E). Interesting differences in the level of the colony formation for untreated and cuprizone-treated mice was observed for CFU-E colonies. While these colonies increased from 0 to 40 days for untreated mice from 3.2 to $6.0 \%$, cuprizone suppressed the number of these colonies to $3.3 \%$ at 40 days (Figure $5 \mathrm{C}$ ). Thus, cuprizone changes the differentiation profile of bone marrow stem cells.

It was reasonable to suggest that there should be a strong increase of precursors analyzed during development of autoimmune processes. Earlier studies of development of spontaneous and DNA-induced SLE in MRL-lpr/lpr mice [32-34] and the findings presented here revealed significant changes in the profiles of stem cell differentiation. Maximal increment of bone marrow precursor cells was observed for CFU-E and CFU-GM colonies (Figure 5). Interestingly, the treatment of mice with cuprizone leads to the suppression of the formation of anti-DNA, anti-MBP, and anti-MOG antibodies as well as to decrease in their catalytic activities (Figure 1 and 3). At the same time, cuprizone decreased significantly only the proliferation of CFU-E cells (Figure 5C), while CFU-GM cells were comparable for untreated and cuprizone-treated mice.

For additional verification of this assumption we have calculated a relative percent of various precursors of MOG-induced EAE animals (Figure 5B and 5E). Interestingly, the concentrations of anti-MOG and anti-DNA Abs from the sera of MOG-treated mice at 22 days is very high (Figure $1 \mathrm{C}$ and $1 \mathrm{D}$ ) and their catalytic activities are maximal (Figures 3C and 3E). However, the mice treatment with MOG leads to the decrease in the percent content of CFU-GM, BFU-E, and CFU-GEMM colonies at 18-22 days of the experiment (Figure 5).

\section{Discussion}

As it was shown previously, the detection of Abs with MBP-hydrolyzing and DNase activities in human and animal's plasma may be considered to be a reliable indicator of the onset or a significant development of autoimmune processes associated with several autoimmune diseases [10-14]. The spontaneous increase in proteinuria and in anti-MOG-, anti-MBP-, and anti-DNA specific auto-Abs in the case of untreated mice shows that C57BL/6 mice are autoimmune-prone prototypical strain, in which the spontaneous development of autoimmune reactions may occur and their state of "health" may be considered as provisional [36]. Increased DNA-, MBP-, and MOG-hydrolyzing activities of IgGs were revealed at a time corresponding to the acute phase of EAE (6-8 days), followed by a statistically significant increase in their activities at 14-22 days (Figure 3). Later, a significant decrease in all activities at the transition from the acute (18-22 days) to the severe chronic phase of EAE (40 days) induced by mice treatment with MOG was observed. Overall, in C57BL/6 mice there is a spontaneous and MOG-induced formation of antibodies hydrolyzing MBP, MOG, and DNA with high activity.

In this article we analyzed for the first time possible changes of a subset of biochemical indexes during EAE development including RAs of abzymes, differentiation profile HSC and lymphocyte proliferation after autoimmune-prone C57BL/6 mice treatment with cuprizone. It was shown that cuprizone only slightly decreased the weight and level of proteinuria of cuprizone-treated comparing with untreated C57BL/6 mice (Figure 1A and 1B). The mice treatment with cuprizone promotes the decrease in the blood concentration of anti-MOG and anti-DNA antibodies (Figure 1C and 1D) and the RAs of these Abs in the hydrolysis of MOG, MBP, and DNA (Figure 3C and 3E) in comparison to those for untreated and especially MOG-treated mice.

We have recently analyzed the variation of various parameters in spontaneous and MOG-induced EAE development in mice [36]. In this study we confirmed previously obtained results. Taking into account the deviation of different parameters described above for of each group in the case of spontaneous and MOG-induced EAE development estimated in this study were not differ from those obtained in [36].

In this study we confirmed previously obtained results; taking into account the deviation of the activity values for seven MOGtreated mice of each group, the average relative activity of Abs in this study were not differ from those obtained in [36].

We try to recognize possible mechanistic differences in the spontaneous development of EAE, its acceleration by treatment of mice with MOG and their immunization with cuprizone. Here we have shown that spontaneous significant progress in mice EAE occurs during 20-40 days of the experiment. However, we found no statistically significant differences in the brain corpus callosum size and appearance of plaques in the brain of untreated mice over time (Figure 4). Chemical stimulation of demyelination led to a statically significant reduction in the area of the brain corpus callosum in cuprizone-treated mice at 20 days and even stronger at 40 days of the experiment (Figure 4). Thus, one can suppose, that demyelination induced by cuprizone is likely not associated with the typical autoimmune processes development observed after spontaneous or MOG-induced EAE. It looks more like as s 
specific mice poisoning with cuprizone, leading to the manifestation of some symptoms typical for EAE. As it was shown above, MOG-induced and spontaneous development of EAE is associated with a specific reorganization of the immune system leading to changes in differentiation of mice bone marrow hematopoietic stem cells and to the increase in blood concentration of anti-MOG and anti-DNA antibodies and their relative enzymatic activities. However, cuprizone does not change significantly the profile of differentiation of BFU-E, CFU-GM, and CFU-GEMM cells (Figure 5). Maximal increase in the level of proliferation during spontaneous and MOG-induced development of EAE was observed only for CFU-E cells (Figure 5). It is important, that cuprizone powerfully inhibits the proliferation of only CFU-E cells (Figure 5).

Demyelination of nerve fibers is a critical process in the formation and functioning of the central and peripheral nervous system [1$5,35]$. The myelin sheath around axons is necessary for efficient conduction of electrical impulses through the nerves. Destruction of myelin occurs in a number of diseases, which include in particular MS. As it was shown earlier, abzymes hydrolyzing MBP may play an important harmful role in MS pathogenesis, since they attack and hydrolyze MBP of the myelin-proteolipid sheath of axons, which lead to demyelination of brain tissue and fiber tracts [22-27]. The treatment of mice with cuprizone also leads to the demyelination of the brain as obtained by MRI (Figure 4).

It is also known, that cuprizone has selective specificity against oligodendrocytes [37,38]. That is why the cuprizone mice model is a widely used experimental paradigm for analysis the myelin pathology in MS. The process of "acute demyelination" in mice is usually associated with activation of microglia and phagocytosis of myelin sheaths and apoptosis of oligodendrocytes [37,38]. The main functions of oligodendrocytes are the support and insulation of axons in the central nervous system of vertebrates. Oligodendrocytes create the myelin sheath consisting of $20 \%$ proteins and $80 \%$ lipids [50]. Each oligodendrocyte forms one myelin segment for several neighboring axons [50]. Taking this into account, one cannot exclude that cuprizone-dependent apoptosis of oligodendrocytes together with the activation of myelin sheaths phagocytosis and microglia will induce pathological immuno processes. Since cuprizone does not have a significant influence on the time course of proliferation of CFU-GM, BFU-E, and CFU-GEMM cells in comparison to untreated mice, there seems to be no significant effect on these cells. However, cuprizone suppresses significantly the proliferation of only CFU-E cells, which is observed for MOG-treated and untreated mice (Figure 5).

It should be noted, that autoimmune prone MRL-lpr/lpr and C57BL/6 are characterized by the spontaneous development of a condition which is susceptible for autoimmune attacks and associated with significant, and in many respects similar changes in the differentiation of bone marrow HSCs, lymphocyte proliferation in different organs, and production of abzymes hydrolyzing DNA [36]. In contrast to MRL-lpr/lpr mice, the induction of EAE in C57BL/6 mice correlates with production of abzymes hydrolyzing MOG and MBP [36]. One cannot exclude, the development of other different autoimmune diseases is also may be associated with significant changes in the HSC differentiation profile and lymphocyte proliferation, which may be an important prime cause for the initial stage of these autoimmune processes.

\section{Conclusion}

Taken together, autoimmune prone C57BL/6 mice inherent to spontaneous development of EAE leading to significant changes in the differentiation of bone marrow HSCs and production of MOG-, MBP-, and DNA-hydrolyzing abzymes, that could be an important root cause of the initial stage of the autoimmune processes.

Obviously, the disease symptoms associated with treatment by cuprizone are associated with demyelination, but not with autoimmune processes.

\section{Acknowledgement}

This research was made possible by grants mainly from Russian Science Foundation (No 16-15-10103 to G. A. Nevinsky) and cooperation with the laboratory in Germany was supported by Bundesministerium für Bildung und Forschung (BMBF, $01 D J 12103$ and DFG, CRC128-B06 to T.B and S.G.M.).

\section{References}

1. O'Connor KC, Bar-Or A, Hafler DA (2001) The neuroimmunology of multiple sclerosis: possible roles of T and B lymphocytes in immunopathogenesis. J Clin Immunol 21: 81-92.

2. Archelos JJ, Storch MK, Hartung HP (2000) The role of B cells and autoantibodies in multiple sclerosis. Ann Neurol 47: 694-706.

3. Hemmer B, Archelos JJ, Hartung HP (2002) New concepts in the immunopathogenesis of multiple sclerosis. Nat Rev Neurosci 3: 291-301.

4. Niehaus A, Shi J, Grzenkowski M, Diers-Fenger M, Archelos J, et al. (2000) Patients with active relapsing-remitting multiple sclerosis synthesize antibodies recognizing oligodendrocyte progenitor cell surface protein: implications for remyelination. Ann Neurol 48: 362-71.

5. Cross AH, Trotter JL, Lyons J (2001) B cells and antibodies in CNS demyelinating disease. J Neuroimmunol 112: 1-14.

6. Lerner RA, Tramontano A (1987) Antibodies as enzymes. Trends Biochem Sci 12: 427-30.

7. Stewart JD, Benkovic SJ (1993) Recent developments in catalytic antibodies. Int Rev Immunol 10: 229-40.

8. Martin AB, Schultz PG (1999) Opportunities at the interface of chemistry and biology. Trends Cell Biol 9: M24-8.

9. Keinan E (2005) Catalytic antibodies, Weinheim, Germany: Wiley-VCH Verlag GmbH and Co. KgaA 1-586.

10. Keinan E (2005) Natural catalytic antibodies-abzymes In: Catalytic antibodies, Wiley-VCH Verlag GmbH \& Co. KGaA 505-69. 
11. Brenner KJ (2010) Natural catalytic antibodies in norm and in autoimmune diseases. In Autoimmune diseases: symptoms, diagnosis and treatment. NY: Nova Science Publishers Inc New York 1-107.

12. Nevinsky GA, Buneva VN (2010) Natural catalytic antibodies in norm, autoimmune, viral, and bacterial diseases. ScientificWorldJournal 10: $1203-33$.

13. Nevinsky GA (2011) Natural Catalytic Antibodies in Norm and in HIV-Infected Patients, Understanding HIV/AIDS Management and Care - Pandemic Approaches in the 21st Century, Dr. Fyson Kasenga Rijeka, Croatia: InTech 151-92.

14. Nevinsky GA, Buneva VN (2012) Autoantibodies and Natural Catalytic Antibodies in Health, Multiple Sclerosis, and Some Other Diseases. Adv Neuroimmune Biol 3: 157-82.

15. Berneman A, Guilbert B, Eschrich S, Avrameas S (1993) IgG auto- and polyreactivities of normal human sera. Mol Immunol 30: 1499-510.

16. Coutinho A, Kazatchkine MD, Avrameas S (1995) Natural autoantibodies. Curr Opin Immunol 7: 812-8.

17. Baranovskii AG, Kanyshkova TG, Mogelnitskii AS, Naumov VA, Buneva VN, et al. (1998) Polyclonal antibodies from blood and cerebrospinal fluid of patients with multiple sclerosis effectively hydrolyze DNA and RNA. Biochemistry 63: 1239-48.

18. Baranovskii AG, Ershova NA, Buneva VN, Kanyshkova TG, Mogelnitskii AS, et al. (2001) Catalytic heterogeneity of polyclonal DNA-hydrolyzing antibodies from the sera of patients with multiple sclerosis. Immunol Lett 76: 163-7.

19. Ivanen DR, Kulminskaya AA, Shabalin KA, Isaeva-Ivanova LV, Ershova NA, et al. (2004) Catalytic properties of IgMs with amylolytic activity isolated from patients with multiple sclerosis. Med Sci Monit 10: BR273-80.

20. Savel'ev AN, Kulminskaya AA, Ivanen DR, Nevinsky GA, et al. (2004) Human antibodies with amylolytic activity. Trends in Glycoscience and Glycotechnology 16: $17-31$

21. Nevinsky GA, Buneva VN (2003) Catalytic antibodies in healthy humans and patients with autoimmune and viral diseases. J Cell Mol Med 7: 265-76.

22. Polosukhina DI, Kanyshkova TG, Doronin BM, Tyshkevich OB, Buneva VN, et al. (2004) Hydrolysis of myelin basic protein by polyclonal catalytic IgGs from the sera of patients with multiple sclerosis. J Cell Mol Med 8: 359-68.

23. Polosukhina DI, Buneva VN, Doronin BM, Tyshkevich OB, Boiko AN, et al. (2005) Hydrolysis of myelin basic protein by IgM and IgA antibodies from the sera of patients with multiple sclerosis. Med Sci Monit 11: BR266-72.

24. Polosukhina DI, Kanyshkova TG, Doronin BM, Tyshkevich OB, Buneva VN, et al. (2006) Metal-dependent hydrolysis of myelin basic protein by IgGs from the sera of patients with multiple sclerosis. Immunol Lett 103: 75-81.

25. Parkhomenko TA, Doronin VB, Castellazzi M, Padroni M, Pastore M, et al. (2014) Comparison of DNA-Hydrolyzing Antibodies from the Cerebrospinal Fluid and Serum of Patients with Multiple Sclerosis. PLoS One 9: e93001.

26. Doronin VB, Parkhomenko TA, Castellazzi M, Padroni M, Pastore M, et al. (2014) Comparison of antibodies hydrolyzing myelin basic protein from the cerebrospinal fluid and serum of patients with multiple sclerosis. PLoS One 9: e107807.

27. Belogurov AA, Kurkova IN, Friboulet A, Thomas D, Misikov VK, et al. (2008) Recognition and degradation of myelin basic protein peptides by serum autoantibodies: novel biomarker for multiple sclerosis. J Immunol 180: 1258-67.

28. Nagata S, Suda T (1995) Fas and Fas ligand: lpr and gld mutations. Immunol Today 16: 39-43.

29. Watanabe-Fukunaga R, Brannan CI, Copeland NG, Jenkins NA, Nagata S (1992) Lymphoproliferation disorder in mice explained by defects in Fas antigen that mediates apoptosis. Nature 356: 314-7.

30. Fournel S, Muller S (2002) Anti-nucleosome antibodies and T-cell response in systemic lupus erythematosus. Ann Med Interne 153: 513-9.

31. Ikehara S, Kawamura M, Takao F, Inaba M, Yasumizu R, et al. (1990) Organ-specific and systemic autoimmune diseases originate from defects in hematopoietic stem cells. Proc Natl Acad Sci U S A 87: 8341-4.

32. Andryushkova AA, Kuznetsova IA, Orlovskaya IA, Buneva VN, Nevinsky GA (2006) Antibodies with amylase activity from the sera of autoimmune-prone MRL/MpJ-lpr mice. FEBS Lett 580: 5089-95.

33. Andryushkova AA, Kuznetsova IA, Orlovskaya IA, Buneva VN, Nevinsky GA (2009) Nucleotide-hydrolyzing antibodies from the sera of autoimmune-prone MRL-lpr/lpr mice. Int Immunol 21: 935-45.

34. Andryushkova AA, Kuznetsova IA, Bineva VN, Toporkova LB, Sakhno LV, et al. (2007) Formation of different abzymes in autoimmune-prone MRL-lpr/lpr mice is associated with changes in colony formation of haematopoietic progenitors. J Cell Mol Med 11: 531-51.

35. Croxford AL, Kurschus FC, Waisman A (2011) Mouse models for multiple sclerosis: historical facts and future implications. Biochim Biophys Acta 1812: 17783.

36. Doronin VB, Parkhomenko TA, Korablev A, Toporkova LB, Lopatnikova JA, et al. (2016) Changes in different parameters, lymphocyte proliferation and hematopoietic progenitor colony formation in EAE mice treated with myelin oligodendrocyte glycoprotein. J Cell Mol Med 20: 81-94.

37. Blakemore WF, Franklin RJ (2008) Remyelination in experimental models of toxin-induced demyelination. Curr Top Microbiol Immunol 318: 193-212.

38. Lassmann H (2007) Experimental models of multiple sclerosis. Rev Neurol 163: 651-5.

39. Komoly S (2005) Experimental demyelination caused by primary oligodendrocyte dystrophy. Regional distribution of the lesions in the nervous system of mice. Ideggyogy Sz 58: 40-3.

40. Stidworthy MF, Genoud S, Suter U, Mantei N, Franklin RJ (2003) Quantifying the early stages of remyelination following cuprizone-induced demyelination. Brain Pathol 13: 329-39.

41. Mouse EAE models. Overview and Model Selection Hooke Laboratories, Inc; 2011-2013.

42. Delarasse C, Smith P, Baker D, Amor S (2013) Novel pathogenic epitopes of myelin oligodendrocyte glycoprotein induce experimental autoimmune encephalomyelitis in C57BL/6 mice. Immunology 140: 456-64.

43. Tselios T, Aggelidakis M, Tapeinou A, Tseveleki V, Kanistras I, et al. (2014) Rational design and synthesis of altered peptide ligands based on human myelin oligodendrocyte glycoprotein 35-55 epitope: inhibition of chronic experimental autoimmune encephalomyelitis in mice. Molecules 19: 17968-84.

44. McDonald CA, Payne NL, Sun G, Clayton DJ, Del Borgo MP, et al. (2014) Single $\beta^{3}$-Amino Acid Substitutions to MOG Peptides Suppress the Development of Experimental Autoimmune Encephalomyelitis. J Neuroimmunol 277: 67-76.

45. Warnecke A, Musunuri S, N'diaye M, Sandalova T, Achour A, et al. (2016) Nitration of MOG Diminishes Its Encephalitogenicity Depending on MHC Haplotype. J Neuroimmunol 303: 1-12. 
46. Zhang L, Guo Y, Xia CQ (2015) Infusion of Sulfosuccinimidyl-4-[N-maleimidomethyl]cyclohexane-1-carboxylate-Conjugated MOG35-55-Coupled Spleen Cells Effectively Prevents and Reverses Experimental Autoimmune Encephalomyelitis in Mice. J Immunol Res $2015: 129682$.

47. Mimura LA, Chiuso-Minicucci F, Fraga-Silva TF, Zorzella-Pezavento SF, França TG, et al. (2016) Association of myelin peptide with vitamin D prevents autoimmune encephalomyelitis development. Neuroscience 317: 130-40.

48. Simmons SB, Pierson ER, Lee SY, Goverman JM (2013) Modeling the heterogeneity of multiple sclerosis in animals. Trends Immunol 34: 410-22.

49. Cheetham SA, Smith AL, Armstrong SD, Beynon RJ, Hurst JL (2009) Limited variation in the major urinary proteins of laboratory mice. Physiol Behav 96: 253-61.

50. Carlson NR (2010) Physiology of Behavior 10th Ed. Allyn and Bacon, Boston, USA.

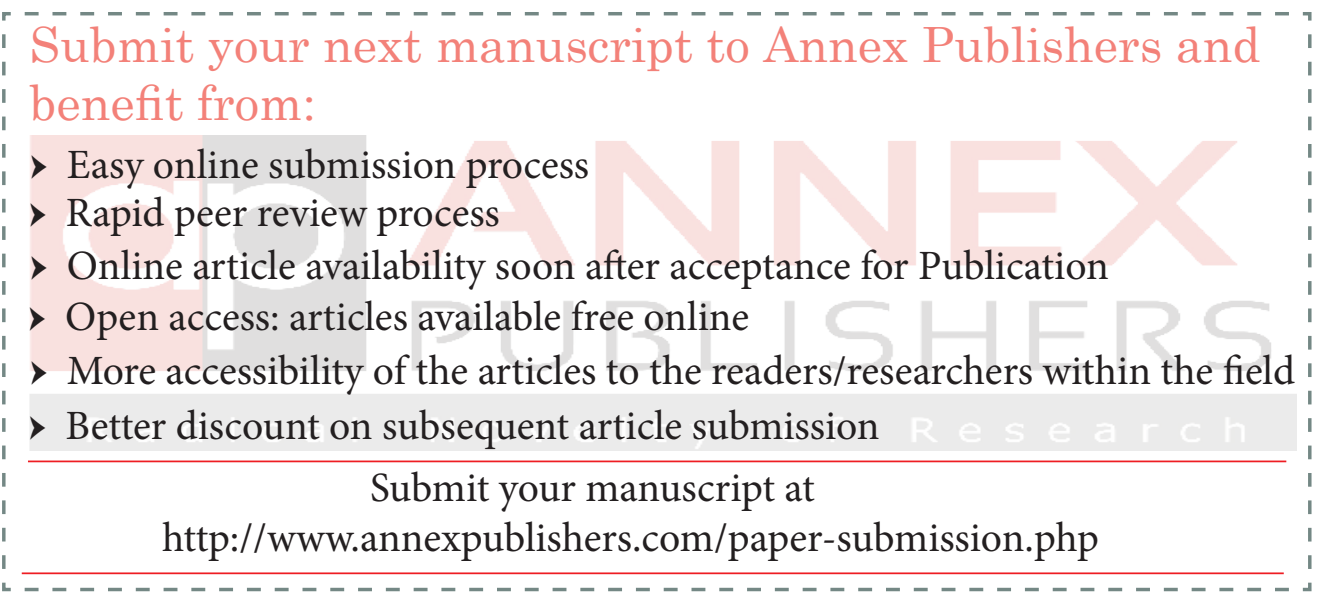

